\title{
A Potential Cyclotron Resonant Scattering Feature in the Ultraluminous X-Ray Source Pulsar NGC 300 ULX1 Seen by NuSTAR and XMM-Newton
}

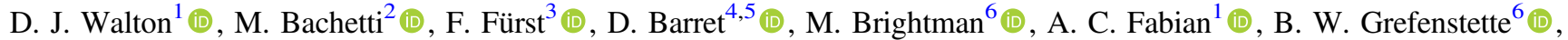

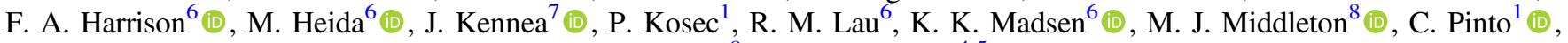 \\ J. F. Steiner ${ }^{9}$ (i), and N. Webb $b^{4,5}$ \\ ${ }^{1}$ Institute of Astronomy, Cambridge University, Madingley Road, Cambridge CB3 0HA, UK \\ ${ }^{2}$ INAF/Osservatorio Astronomico di Cagliari, via della Scienza 5, I-09047 Selargius (CA), Italy \\ ${ }^{3}$ European Space Astronomy Centre (ESA/ESAC), Operations Department, Villanueva de la Cañada (Madrid), Spain \\ ${ }^{4}$ Universite de Toulouse; UPS-OMP; IRAP; Toulouse, France \\ ${ }^{5}$ CNRS; IRAP; 9 Av. colonel Roche, BP 44346, F-31028 Toulouse cedex 4, France \\ ${ }^{6}$ Cahill Center for Astrophysics, California Institute of Technology, 1216 East California Boulevard, Pasadena, CA 91125, USA \\ ${ }^{7}$ Department of Astronomy and Astrophysics, Pennsylvania State University, 525 Davey Lab, University Park, PA 16802, USA \\ ${ }^{8}$ Department of Physics and Astronomy, University of Southampton, Highfield, Southampton SO17 1BJ, UK \\ ${ }^{9}$ MIT Kavli Institute for Astrophysics and Space Research, MIT, 70 Vassar Street, Cambridge, MA 02139, USA \\ Received 2018 March 20; revised 2018 March 27; accepted 2018 March 29; published 2018 April 9
}

\begin{abstract}
Based on phase-resolved broadband spectroscopy using XMM-Newton and NuSTAR, we report on a potential cyclotron resonant scattering feature (CRSF) at $E \sim 13 \mathrm{keV}$ in the pulsed spectrum of the recently discovered ultraluminous X-ray source (ULX) pulsar NGC 300 ULX1. If this interpretation is correct, the implied magnetic field of the central neutron star is $B \sim 10^{12} \mathrm{G}$ (assuming scattering by electrons), similar to that estimated from the observed spin-up of the star, and also similar to known Galactic X-ray pulsars. We discuss the implications of this result for the connection between NGC 300 ULX1 and the other known ULX pulsars, particularly in light of the recent discovery of a likely proton cyclotron line in another ULX, M51 ULX-8.
\end{abstract}

Key words: stars: neutron - X-rays: binaries - X-rays: individual (NGC 300 ULX1)

\section{Introduction}

Ultraluminous X-ray sources (ULXs) are off-nuclear sources that appear to radiate in excess of the Eddington limit for the standard $\sim 10 M_{\odot}$ stellar remnant black holes seen in Galactic $\mathrm{X}$-ray binaries (i.e., $L_{\mathrm{X}}>10^{39} \mathrm{erg} \mathrm{s}^{-1}$; see Kaaret et al. 2017 for a recent review). The discovery that at least some ULXs are powered by accreting pulsars brought about a paradigm shift in our understanding of this exotic population. These neutron stars are the most extreme persistent accretors known, with apparent luminosities up to $\sim 500$ times the Eddington limit for a standard neutron star mass of $1.4 M_{\odot}\left(\sim 2 \times 10^{38} \mathrm{erg} \mathrm{s}^{-1}\right)$. Until recently, only three such sources were known: M82 X-2 (Bachetti et al. 2014), NGC 7793 P13 (Fürst et al. 2016; Israel et al. 2017b), and NGC 5907 ULX1 (Israel et al. 2017a). In addition, although pulsations have not been seen from this source, Brightman et al. (2018) report the likely detection of a cyclotron resonant scattering feature (CRSF) in the spectrum of a ULX in M51, which would require this to be another neutron star accretor. However, the similarity of these sources to the broader ULX population has led to speculation that neutron star accretors may dominate the demographics of ULXs (Koliopanos et al. 2017; Pintore et al. 2017; Walton et al. 2018a, 2018b). The recent discovery of a fourth ULX pulsar in NGC 300 by Carpano et al. (2018) provides further evidence that neutron stars may be common among ULXs.

NGC 300 ULX1 was originally identified as a supernova candidate in 2010 after a strong optical outburst, and given the identifier SN 2010 da (Monard 2010). However, the discovery of a moderately bright $\left(L_{\mathrm{X}} \sim 10^{38} \mathrm{erg} \mathrm{s}^{-1}\right)$, recurring X-ray counterpart and its subsequent rebrightening in the infrared $\sim 2000$ days after the optical outburst ultimately helped to confirm NGC 300 ULX1 to be a high-mass X-ray binary
(Binder et al. 2016; Lau et al. 2016; Villar et al. 2016). Observations in 2016 December revealed a further X-ray outburst from this source, in which it reached observed ULX luminosities $\left(L_{\mathrm{X}} \sim 3 \times 10^{39} \mathrm{erg} \mathrm{s}^{-1}\right.$; Carpano et al. 2018), and the detection of a $\sim 32$-s pulse period from this epoch confirmed the accretor as a neutron star (and the fourth ULX pulsar). Subsequent X-ray monitoring revealed the neutron star to be undergoing an extreme rate of spin-up, with $\dot{P}>10^{-7} \mathrm{~s} / \mathrm{s}$, resulting in a pulse period of $\sim 20 \mathrm{~s}$ only $\sim 15$ months after the 2016 observations in which the pulsations were initially discovered (Bachetti et al. 2018; Kennea 2018). Similar to the other ULX pulsars, the pulse profile appears to be broad and relatively sinusoidal (Carpano et al. 2018), but one of the remarkable aspects of this particular source is that its pulsed fraction is extremely high, reaching $>75 \%$ at energies $>2 \mathrm{keV}$. Finally, there is also now evidence that this source is launching an extreme and variable X-ray wind (Kosec et al. 2018b), consistent with the basic expectation for super-Eddington accretion (Shakura \& Sunyaev 1973; Poutanen et al. 2007) and similar to other ULXs (Pinto et al. 2016; Walton et al. 2016a; Kosec et al. 2018a).

Here we present a broadband X-ray spectral analysis of NGC 300 ULX1, in which we find potential evidence for a CRSF in the 2016 XMM-Newton+NuSTAR data.

\section{Observations and Data Reduction}

NuSTAR (Harrison et al. 2013) and XMM-Newton (Jansen et al. 2001) performed a coordinated observation of the galaxy NGC 300 starting on 2016 December 17 (XMM-Newton OBSIDs 0791010101 and 0791010101, taken over back-toback orbits, and NuSTAR OBSID 30202035002). Although the primary target of the observation was the Wolf-Rayet X-ray 

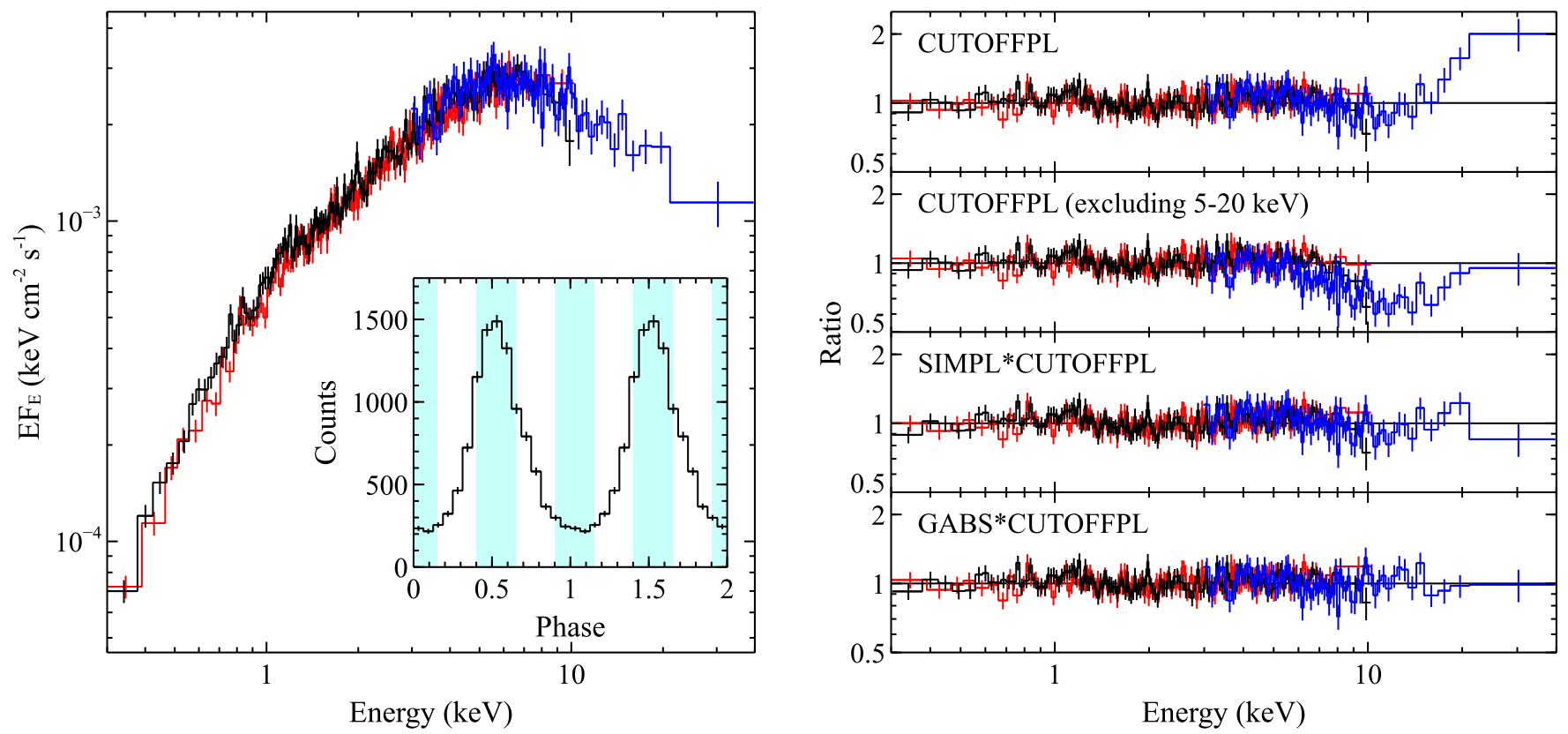

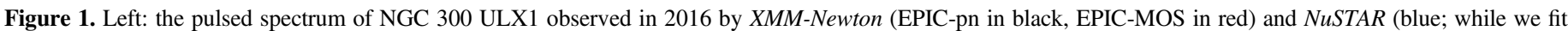

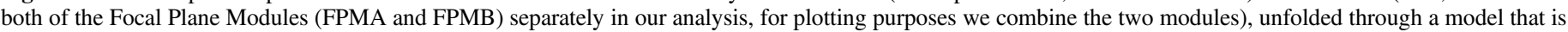

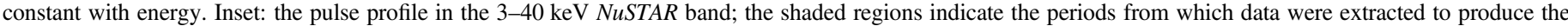
pulsed spectrum in the main panel. Right: data/model ratios for some of the models considered (see Section 3). The data have been rebinned for visual clarity.

binary NGC $300 \mathrm{X}-1$, the observation serendipitously caught an outburst of NGC 300 ULX1. The two sources are separated by $\sim 70^{\prime \prime}$, and during these observations ULX1 is more than an order of magnitude brighter than $\mathrm{X}-1$. Our reduction of the NuSTAR data follows the procedure outlined in Kosec et al. (2018b), and we extract both the standard "science" and the "spacecraft science" data (Walton et al. 2016b). For XMMNewton we just focus on the data from the EPIC-pn and EPICMOS detectors (Strüder et al. 2001; Turner et al. 2001). Our reduction again largely follows Kosec et al. (2018b), although we use a slightly smaller region of $30^{\prime \prime}$ radius to extract source products to reduce the background. The total good exposures are 140 and $190 \mathrm{ks}$ for the EPIC-pn and EPIC-MOS detectors, and $180 \mathrm{ks}$ for the $N u S T A R$ FPMA/B modules, respectively. In all cases, the cleaned event files were barycentered using the DE-200 ephemeris.

\section{The Pulsed Spectrum of NGC 300 ULX1}

Our analysis focuses on the broadband X-ray spectrum of the pulsed emission from the accretion column in NGC 300 ULX1. Model fits are performed with XSPEC v12.6.0f (Arnaud et al. 1996), and unless otherwise stated uncertainties on the spectral parameters are quoted at the $90 \%$ confidence level for one interesting parameter. All of the models presented include a Galactic absorption component with a fixed column of $N_{\mathrm{H}, \mathrm{Gal}}=4.2 \times 10^{20} \mathrm{~cm}^{-2}$ (Kalberla et al. 2005), and we also allow for absorption intrinsic to the source at the redshift of NGC $300\left(N_{\mathrm{H} ; \text { int }} ; z=0.00048\right)$. Both absorption components are modeled with the TBNEW absorption code, and we use the cross-sections of Verner et al. (1996) and the abundance set presented in Wilms et al. (2000). We also allow for crosscalibration uncertainties between the different detectors by including multiplicative constants that are allowed to float between the data sets, fixing Focal Plane Module A (FPMA) at unity. These are always within $\sim 12 \%$ of unity, as expected (Madsen et al. 2015).
We isolate the spectrum of the pulsed component following the approach taken in our recent analyses of M82 X-2, NGC 7793 P13, and NGC 5907 ULX1 (Brightman et al. 2016; Walton et al. 2018a, 2018b). In brief, we phase-tag the cleaned event files, and extract spectra from the brightest and the faintest quarters of the pulse cycle (the pulse profile of NGC 300 ULX1 is broad and nearly sinusoidal, fairly similar to the other known ULX pulsars; see Figure 1). We then subtract the latter from the former (i.e., "pulse-on"-“pulse-off"). The timing solution used to phase-tag the events combines a pulse frequency of $\nu=0.0315275(3) \mathrm{Hz}$ (at MJD 57738.65732) and a strong frequency derivative of $\dot{\nu}=5.535(2) \times 10^{-10} \mathrm{~Hz} \mathrm{~s}^{-1}$ (the parentheses indicate the $1 \sigma$ error on the last digit). This was derived using a combination of the HENDRICS (version 0.4rc1; Bachetti 2015) and PINT $^{10}$ software packages, and is consistent with Carpano et al. (2018). The spectra extracted are rebinned to have a minimum of 25 counts per energy bin to allow the use of $\chi^{2}$ statistics, and we fit the data over the $\sim 0.3-40 \mathrm{keV}$ energy range.

The pulsed spectrum is shown in Figure 1 (left panel). We initially fit these data with a CUTOFFPL model, which provides an excellent description of the pulsed emission in the other ULX pulsars. However, we find that this simple model cannot successfully fit the data for NGC 300 ULX1. Although the fit is not terrible in a statistical sense, with $\chi^{2}=1669$ for 1597 degrees of freedom (DoF), the model leaves a clear excess of emission at the highest energies; the data/model ratios for a number of the models considered here are also shown in Figure 1 (right panel). This is reminiscent of the hard excesses ubiquitously seen in the average spectra of the broadband ULX sample (Walton et al. 2018a), including the ULX pulsars NGC 7793 P13 and NGC 5907 ULX1. However, a critical difference is that in the ULX pulsars (and potentially the rest of the ULX population as well) these excesses in the

\footnotetext{
${ }^{10}$ https://github.com/nanograv/pint
} 
Table 1

Best-fit Parameters Obtained for the Pulsed Spectrum with the Models Including a High-energy Power-law Tail

\begin{tabular}{|c|c|c|}
\hline \multirow{2}{*}{ Parameter } & \multicolumn{2}{|c|}{ Model Combination } \\
\hline & SIMPL $\otimes$ CUTOFFPL & SIMPL $\otimes$ FDCUT \\
\hline$N_{\mathrm{H} ; \text { int }}\left(10^{20} \mathrm{~cm}^{-2}\right)$ & $2.3_{-1.1}^{+1.0}$ & $7.6_{-1.3}^{+1.1}$ \\
\hline$\Gamma$ & $0.72_{-0.05}^{+0.06}$ & $1.20_{-0.06}^{+0.04}$ \\
\hline$E_{\text {cut }}(\mathrm{keV})$ & $\ldots$ & $5.9_{-0.4}^{+1.1}$ \\
\hline$E_{\text {fold }}(\mathrm{keV})$ & $4.9_{-0.4}^{+0.5}$ & $0.8 \pm 0.5$ \\
\hline Norm $\left(10^{-3}\right)$ & $1.7_{-0.6}^{+0.3}$ & $0.8_{-0.2}^{+0.7}$ \\
\hline$\Gamma_{\text {SIMPL }}$ & $<2.8$ & $2.7_{-0.3}^{+0.1}$ \\
\hline$f_{\mathrm{sc}}(\%)$ & $43_{-39}^{+9}$ & $>52$ \\
\hline$\chi^{2} / \mathrm{DoF}$ & $1654 / 1595$ & $1588 / 1594$ \\
\hline
\end{tabular}

average spectra are related to the presence of the pulsed emission from the accretion column (Walton et al. 2018a, $2018 b)$. Here, we have already isolated this pulsed emission.

Nevertheless, it is possible that the pulsed continuum in this particular case is more complex than a simple CUTOFFPL model can describe. We first attempt to fit these data with other phenomenological models typically used for the high-energy emission in neutron star X-ray binaries, which allow for more complex spectral shapes: FDCUT (a cutoff power-law model which includes both the folding energy, $E_{\text {fold }}$, and the energy at which this folding begins to act, $E_{\text {cut }}$, as free parameters; Tanaka 1986) and NPEX (a combination of two CUTOFFPL models with $E_{\text {fold }}$ linked between the two; traditionally the slope of one component is free to vary, while the other is fixed to $\Gamma=-2$; Mihara et al. 1998). However, we find that both of these models result in hard excesses similar to the CUTOFFPL model (and the fits are statistically similar, with $\chi^{2} /$ DoF $=$ $1672 / 1596$ and 1663/1596 for FDCUT and NPEX, respectively). The curvature in the $\sim 5-10 \mathrm{keV}$ band is too strong for any smoothly varying single-component model to account for the highest energies probed by NuSTAR, and a second model component is clearly required to fit the data.

Similar to our early work on the average ULX spectra, we first attempt to account for this high-energy excess by allowing for a further high-energy power-law tail using an additional SIMPL component (Steiner et al. 2009; note that this model has a lower limit on the photon index of $\Gamma_{\text {SIMPL }} \geqslant 1$ ). In these subsequent fits, we find that for the NPEX model the normalization of the $\Gamma=-2$ runs to zero, making the model indistinguishable from the simpler CUTOFFPL case, so from this point we only report the results from the latter. The quality of fit depends on the choice of continuum model (the results are presented in Table 1), but in both of the cases considered the addition of the SIMPL component provides a significant improvement $\left(\Delta \chi^{2} \geqslant 15\right.$ for two additional free parameters, giving a chance improvement probability of 0.004 according to the Akaike Information Criterion), and the high-energy data are much better described (see Figure 1). However, when treating the hard excess as a power-law tail, the sharper curvature that FDCUT can provide in the $\sim 5-10 \mathrm{keV}$ band is preferred.

However, given that there is currently no evidence for similar hard excesses in the pulsed emission from the other ULX pulsars, we also explore another possibility in which the residuals seen in Figure 1 are not caused by a real hard excess, but are actually an indication of a broad cyclotron line causing a deficit in the $\sim 5-20 \mathrm{keV}$ band (see Figure 1). We therefore
Table 2

Best-fit Parameters Obtained for the Pulsed Spectrum with the Models Including a Gaussian CRSF

\begin{tabular}{lcc}
\hline \hline Parameter & \multicolumn{2}{c}{ Model Combination } \\
\cline { 2 - 3 } & GABS $\times$ CUTOFFPL & GABS $\times$ FDCUT \\
\hline$N_{\mathrm{H} ; \text { int }}\left(10^{20} \mathrm{~cm}^{-2}\right)$ & $4.0 \pm 1.0$ & $6.3_{-1.3}^{+1.1}$ \\
$\Gamma$ & $0.88 \pm 0.04$ & $1.11_{-0.08}^{+0.05}$ \\
$E_{\text {cut }}(\mathrm{keV})$ & $\ldots$ & $11.2_{-7.1}^{+5.5}$ \\
$E_{\text {fold }}(\mathrm{keV})$ & $7.4_{-0.6}^{+0.7}$ & $4.9_{-1.8}^{+1.2}$ \\
Norm $\left(10^{-3}\right)$ & $0.96 \pm 0.04$ & $1.0_{-1.2}^{+0.5}$ \\
$E_{\mathrm{CRSF}}(\mathrm{keV})$ & $12.8_{-0.9}^{+1.0}$ & $12.8_{-0.9}^{+1.1}$ \\
$\sigma_{\mathrm{CRSF}}(\mathrm{keV})$ & $3.1_{-0.7}^{+0.8}$ & $3.9_{-0.9}^{+1.1}$ \\
$d_{\mathrm{CRSF}}$ & $3.5_{-1.2}^{+1.7}$ & $7.6_{-3.5}^{+8.8}$ \\
\hline$\chi^{2} / \mathrm{DoF}$ & $1607 / 1594$ & $1593 / 1593$ \\
\hline
\end{tabular}

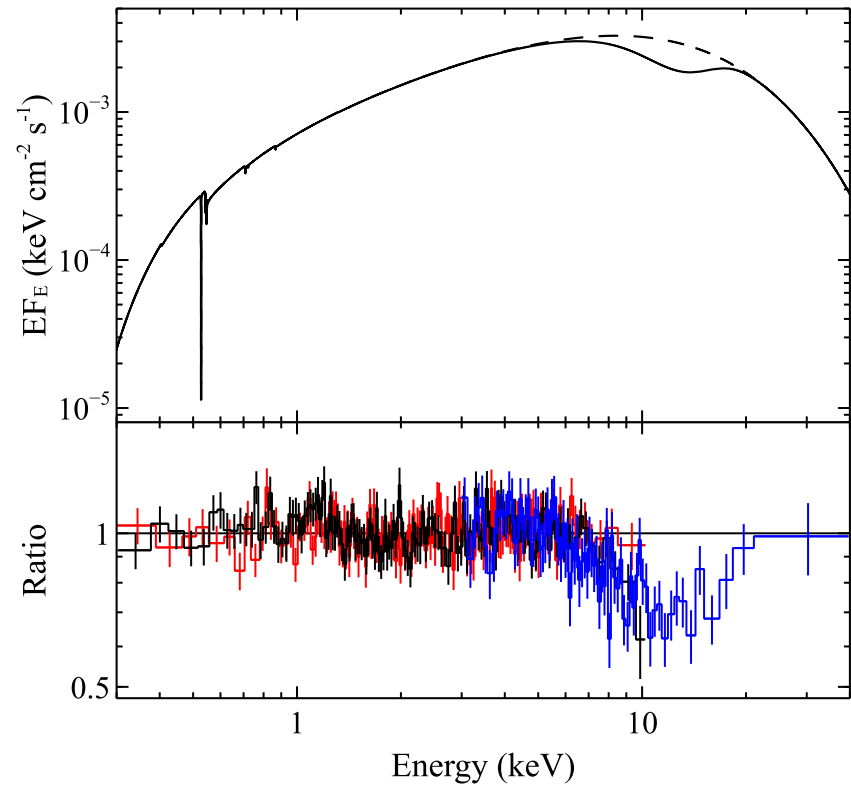

Figure 2. Top: the best-fit model combining GABS $\times$ CUTOFFPL for the pulsed spectrum of NGC 300 ULX1. The solid line shows the full model, and the dashed line shows the intrinsic continuum after removal of the CRSF. Bottom: data/model ratio after the removal of the CRSF, showing the best-fit line profile. This agrees well with that inferred in Figure 1 (the same data are shown).

replace SIMPL with GABS, which provides a multiplicative line with a Gaussian optical depth profile (energy $E$, width $\sigma$, depth $d$ ) and is often used to describe CRSF features (e.g., Staubert et al. 2007; Fürst et al. 2014a, 2014b; Jaisawal $\&$ Naik 2016). For both of the continuum models presented, this provides a similarly excellent description of the data, and a significant improvement over the baseline continuum fits $\left(\Delta \chi^{2} \geqslant 60\right.$ for three additional parameters; Table 2$)$. We show the line profile inferred with the CUTOFFPL continuum in Figure 2. The line parameters are consistent for the two continuum models (see Figure 3), and there is particularly good agreement over a line energy of $E_{\mathrm{CRSF}}=12.8 \mathrm{keV}$. In the case of the CUTOFFPL continuum, the CRSF provides a superior fit to the broadband data than the model including SIMPL, while for the FDCUT continuum the CRSF and SIMPL fits are statistically very similar. We also show the contours of the baseline continuum parameters for the CUTOFFPL model for comparison with the other ULX pulsars in Figure 3; aside from 

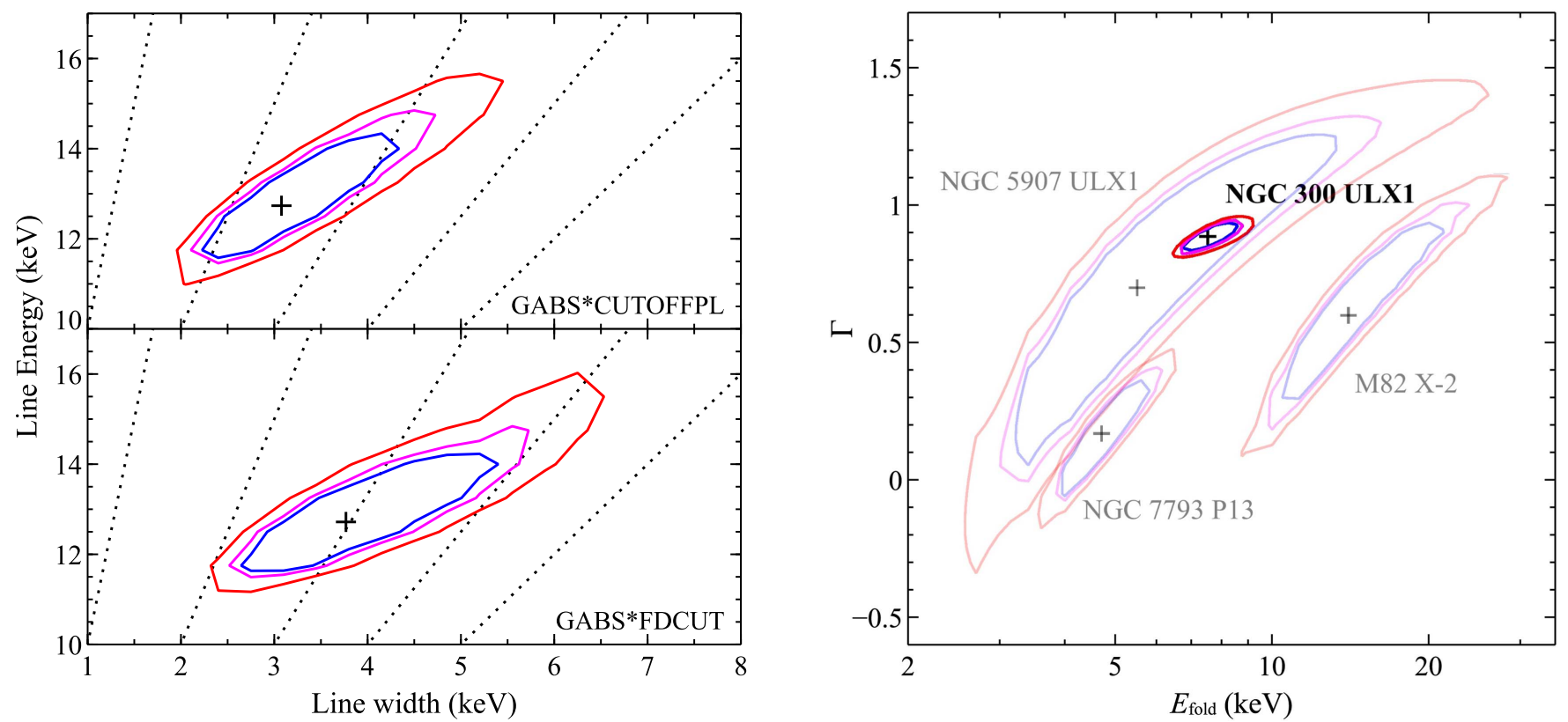

Figure 3. Left: confidence contours for the line energy and width for the CUTOFFPL (top) and FDCUT (bottom) continuum models. The dotted lines show $\sigma_{\mathrm{CRSF}} / E_{\mathrm{CRSF}}=0.1,0.2,0.3,0.4$ and 0.5 (from left to right). Right: a comparison of the constraints on $\Gamma$ and $E_{\text {fold }}$ for the pulsed spectrum of NGC 300 ULX1 with the GABS*CUTOFFPL (solid contours) model with the constraints for the other known ULX pulsars (transparent contours). In each case, the $90 \%$, 95\%, and 99\% confidence contours for two parameters of interest are shown in blue, magenta, and red, respectively.

the additional complexity in the 5-20 keV band, the pulsed spectrum of NGC 300 ULX1 appears broadly similar to that seen from M82 X-2, NGC 7793 P13, and NGC 5907 ULX1.

\section{Discussion and Conclusions}

The pulsed spectrum from the 2016 XMM-Newton $+N u S T A R$ observation of the ULX pulsar NGC 300 ULX1-the latest addition to the sample of ULX pulsars (Carpano et al. 2018) cannot be fit with simple, single-component continuum models. The curvature in the $\sim 5-15 \mathrm{keV}$ band is quite strong, and there is more emission above $\sim 20 \mathrm{keV}$ than can be accounted for by any smoothly varying model that adequately fits this curvature. In addition to a curved baseline continuum, the data can be well explained with either an additional high-energy power-law tail, or a cyclotron scattering feature at $E_{\mathrm{CRSF}}=12.8 \mathrm{keV}$ imprinted on top of an otherwise simple continuum. The other known ULX pulsars do not currently show any evidence for a hard excess in their pulsed spectra that could also be related to the presence of a high-energy power-law tail (Brightman et al. 2016; Walton et al. 2018a, 2018b). In addition, when invoking an additional power-law tail, $E_{\text {fold }}$ is abnormally low when compared with Galactic X-ray pulsars for the FDCUT model preferred in this scenario (e.g., Fürst et al. 2014b; Vybornov et al. 2017). Therefore, although we cannot rule out the possibility that the intrinsic pulsed continuum is more complex in this case, we prefer the CRSF interpretation, and will focus our discussion on this solution.

The potential detection of a CRSF is significant, as these features offer the most robust measure of the magnetic field $(B)$ close to the surface of the neutron star, as the energy of the fundamental electron CRSF (in $\mathrm{keV}$ ) is given by $E_{\mathrm{CRSF}}=$ $11.57 \times B_{12}\left(1+z_{\text {grav }}\right)$, assuming electron scattering. Here, $B_{12}$ is the magnetic field strength in units of $10^{12} \mathrm{G}$, and $z_{\text {grav }}$ is the gravitational redshift of the line-forming region. Assuming that the potential CRSF is indeed the fundamental, this solution would therefore imply $B \sim 10^{12} \mathrm{G}$ (as $z_{\text {grav }} \leqslant 0.25$, as the line must be formed at or beyond the neutron star surface). Critically, this is remarkably similar to the B-field estimated from the spin-up of NGC 300 ULX1 $\left(B \sim 3 \times 10^{12} \mathrm{G}\right.$; Carpano et al. 2018), particularly given the uncertainties in both of these calculations (the exact position of the lineforming region is not formally well known (Poutanen et al. 2013) and because $B \propto(\dot{P} / P)^{7 / 2}$ any errors on $P$ and $\dot{P}$ are magnified when estimating $B$ with this method). This is also quite similar to the magnetic fields inferred for typical Galactic X-ray pulsars (see Caballero \& Wilms 2012 for a review). Furthermore, the width of the line inferred in NGC 300 ULX1 relative to its energy is similar to the electron CRSF (eCRSF) features seen in other luminous X-ray pulsars (where such features have been detected). Both V0332+53 and SMC X-2 show $\sigma_{\mathrm{CRSF}} / E_{\mathrm{CRSF}} \sim 0.2$ (Tsygankov et al. 2006; Jaisawal \& Naik 2016), and for NGC 300 ULX1 we find $0.2 \lesssim \sigma_{\mathrm{CRSF}} / E_{\mathrm{CRSF}} \lesssim 0.4$ (combining the constraints for the continuum models considered; see Figure 3). As an aside, we note that because the line is broad, it should not significantly impact the detection of the wind reported by Kosec et al. (2018b).

The only other probable CRSF reported from a ULX to date comes from M51 ULX-8, where Brightman et al. (2018) report a narrow $(\sigma<0.2 \mathrm{keV})$ absorption feature at $4.5 \mathrm{keV}$. Although a firm identification is not possible, the preferred interpretation for M51 ULX-8 is a proton CRSF (pCRSF), rather than an eCRSF. If correct, this would imply an extreme (magnetar-like) magnetic field of $B \sim 7 \times 10^{14} \mathrm{G}$. This identification was based on the ratio of the width and energy of the line, $\sigma_{\mathrm{CRSF}} / E_{\mathrm{CRSF}}<0.045$, which is unusually low in comparison to the eCRSF features seen in other luminous pulsars (see above), but is more in line with the pCRSF features claimed in some magnetar spectra (Ibrahim et al. 2002; Tiengo et al. 2013). Thus, the similarity of $\sigma_{\mathrm{CRSF}} / E_{\mathrm{CRSF}}$ between NGC 300 ULX1 and the other high-luminosity pulsars further strengthens the pCRSF interpretation for M51 ULX-8 
(although the inclination effects discussed by Meszaros \& Nagel 1985 mean that it is still difficult to completely exclude an eCRSF).

Magnetar-level B-fields are one of the possibilities invoked to explain the extreme luminosities seen from the ULX pulsars known prior to NGC 300 ULX1 (e.g., Mushtukov et al. 2015, and references therein). Fields this strong suppress the electron scattering cross-section (Herold 1979), which locally reduces the radiation pressure and raises the effective Eddington limit. In NGC 300 ULX1, which has a peak luminosity of $L_{\mathrm{X} \text {,peak }} \sim$ $3 \times 10^{39} \mathrm{erg} \mathrm{s}^{-1}$, the inferred B-field $\left(B \sim 10^{12} \mathrm{G}\right)$ is too weak for significant suppression of the cross-section to occur. However, M51 ULX-8 has both a much stronger field $(B \sim$ $\left.7 \times 10^{14} \mathrm{G}\right)$ and a higher peak luminosity of $L_{\mathrm{X} \text {,peak }} \sim$ $10^{40} \mathrm{erg} \mathrm{s}^{-1}$. This is similar to the other known ULX pulsars, which also have $L_{X \text {,peak }} \geqslant 10^{40} \mathrm{erg} \mathrm{s}^{-1}, \sim 100 \times$ (or more) the classical Eddington limit for a standard neutron star (i.e., assuming the Thomson cross-section). It is important to note that the cross-section can only be suppressed for electrons in the regions of strong magnetic field, i.e., the accretion column. Phase-resolved analysis of the ULX pulsars NGC 7793 P13 and NGC 5907 ULX1 implies there are additional, non-pulsed components that likely arise from the accretion flow beyond the magnetospheric radius $\left(R_{\mathrm{M}}\right)$ and can make a significant contribution to the total X-ray flux (up to $\sim 50 \%$; Walton et al. 2018a, 2018b). These cannot be subject to the same magnetic effects, which means that there is a limit to how much the B-field can help to increase the total luminosity in these cases, and that super-Eddington accretion is still required in addition to any magnetic effects (see also King et al. 2017). Nevertheless, taken at face value, the results for M51 ULX-8 and NGC 300 ULX1 are consistent with the idea that in the more luminous ULXs very strong B-fields help to boost the observed luminosity to some degree, but this does not occur in the less luminous systems.

Assuming that M82 X-2, NGC 7793 P13, and NGC 5907 ULX1 do host magnetar-level fields similar to M51 ULX-8, given their spin periods of $\sim 1 \mathrm{~s}$ it has been suggested that these fields may have to be quadrupolar in nature in order to prevent these sources from being persistently in the propeller regime (which is clearly not the case; Israel et al. 2017a). If the difference in B-field strength between NGC 300 ULX1 and the other ULX pulsars is driven by the absence of a strong quadrupole B-field component in the former, this may also help to explain the much higher pulse fractions seen in NGC 300 ULX1, even at the highest energies probed by $N u S T A R$ (where dilution from the accretion flow beyond $R_{\mathrm{M}}$ is negligible; $>70 \%$ in NGC 300 ULX1 versus $\sim 30 \%$ in the other ULX pulsars), as quadrupolar field geometries can result in significantly diluted pulse fractions (Long et al. 2008). Significantly stronger fields in M82 X-2, NGC 7793 P13, and NGC 5907 ULX1 would also naturally explain the lack of evidence for similar CRSFs in their pulsed spectra, as they would be outside of the currently observable band.

Observationally confirming that the complexity in the pulsed spectrum of NGC 300 ULX1 arises from a CRSF is clearly of significant importance. Distinguishing between the high-energy power-law tail and CRSF solutions may require extending the coverage of the high-energy continuum to energies above $40 \mathrm{keV}$, where the two models naturally diverge, or may be possible by identifying the expected luminosity-dependent variations in $E_{\mathrm{CRSF}}$ (e.g., Fürst et al. $2014 \mathrm{~b}$ and references therein). Unfortunately, based on the current peak flux exhibited by NGC 300 ULX1, the former will be extremely challenging for current facilities (i.e., NuSTAR), and may require the next generation of hard X-ray observatory such as the High Energy X-ray Probe, ${ }^{11}$ a potential successor to NuSTAR.

The authors would like to thank the reviewer for timely and positive feedback. D.J.W. and M.J.M. acknowledge support from STFC Ernest Rutherford fellowships. A.C.F. acknowledges ERC Advanced Grant 340442, P.K. acknowledges support from the STFC, and D.B. acknowledges support from the French Space Agency (CNES). This research has made use of data obtained with $N u S T A R$, a project led by Caltech, funded by NASA and managed by NASA/JPL, and has utilized the NUSTARDAS software package, jointly developed by the ASDC (Italy) and Caltech (USA). This work has also made use of data obtained with XMM-Newton, an ESA science mission with instruments and contributions directly funded by ESA Member States.

Facilities: NuSTAR, XMM.

\section{ORCID iDs}

D. J. Walton (1D https://orcid.org/0000-0001-5819-3552 M. Bachetti (iD https://orcid.org/0000-0002-4576-9337

F. Fürst (i) https://orcid.org/0000-0003-0388-0560

D. Barret (iD https://orcid.org/0000-0002-0393-9190

M. Brightman (i) https://orcid.org/0000-0002-8147-2602

A. C. Fabian (iD https://orcid.org/0000-0002-9378-4072

B. W. Grefenstette (iD https://orcid.org/0000-0002-1984-2932

F. A. Harrison (1) https://orcid.org/0000-0003-2992-8024

M. Heida (1) https://orcid.org/0000-0002-1082-7496

J. Kennea (iD https://orcid.org/0000-0002-6745-4790

K. K. Madsen (i) https://orcid.org/0000-0003-1252-4891

M. J. Middleton (ib https://orcid.org/0000-0002-8183-2970

C. Pinto (1) https://orcid.org/0000-0003-2532-7379

J. F. Steiner (iD https://orcid.org/0000-0002-5872-6061

\section{References}

Arnaud, K. A. 1996, in ASP Conf. Ser. 101, Astronomical Data Analysis Software and Systems V, ed. G. H. Jacoby \& J. Barnes (San Francisco, CA: ASP), 17

Bachetti, M. 2015, MaLTPyNT: Quick look timing analysis for NuSTAR data, Astrophysics Source Code Library, ascl:1502.021

Bachetti, M., Grefenstette, B. W., Walton, D. J., et al. 2018, ATel, 11282, 1 Bachetti, M., Harrison, F. A., Walton, D. J., et al. 2014, Nat, 514, 202

Binder, B., Williams, B. F., Kong, A. K. H., et al. 2016, MNRAS, 457, 1636 Brightman, M., Harrison, F., Walton, D. J., et al. 2016, ApJ, 816, 60 Brightman, M., Harrison, F. A., Fürst, F., et al. 2018, NatAs, 2, 312 Caballero, I., \& Wilms, J. 2012, MmSAI, 83, 230

Carpano, S., Haberl, F., Maitra, C., \& Vasilopoulos, G. 2018, MNRAS, 476, 45 Fürst, F., Pottschmidt, K., Wilms, J., et al. 2014a, ApJL, 784, L40

Fürst, F., Pottschmidt, K., Wilms, J., et al. 2014b, ApJ, 780, 133

Fürst, F., Walton, D. J., Harrison, F. A., et al. 2016, ApJL, 831, L14

Harrison, F. A., Craig, W. W., Christensen, F. E., et al. 2013, ApJ, 770, 103 Herold, H. 1979, PhRvD, 19, 2868

Ibrahim, A. I., Safi-Harb, S., Swank, J. H., et al. 2002, ApJL, 574, L51

Israel, G. L., Belfiore, A., Stella, L., et al. 2017a, Sci, 355, 817

Israel, G. L., Papitto, A., Esposito, P., et al. 2017b, MNRAS, 466, L48

Jaisawal, G. K., \& Naik, S. 2016, MNRAS, 461, L97

Jansen, F., Lumb, D., Altieri, B., et al. 2001, A\&A, 365, L1

Kaaret, P., Feng, H., \& Roberts, T. P. 2017, ARA\&A, 55, 303

Kalberla, P. M. W., Burton, W. B., Hartmann, D., et al. 2005, A\&A, 440, 775 Kennea, J. A. 2018, ATel, 11229, 1

$\overline{11}$ https://pcos.gsfc.nasa.gov/physpag/probe/HEXP_2016.pdf 
King, A., Lasota, J.-P., \& Kluźniak, W. 2017, MNRAS, 468, L59

Koliopanos, F., Vasilopoulos, G., Godet, O., et al. 2017, A\&A, 608, A47

Kosec, P., Pinto, C., Fabian, A. C., \& Walton, D. J. 2018a, MNRAS, 473, 5680

Kosec, P., Pinto, C., Walton, D. J., et al. 2018b, arXiv:1803.02367

Lau, R. M., Kasliwal, M. M., Bond, H. E., et al. 2016, ApJ, 830, 142

Long, M., Romanova, M. M., \& Lovelace, R. V. E. 2008, MNRAS, 386, 1274

Madsen, K. K., Harrison, F. A., Markwardt, C. B., et al. 2015, ApJS, 220, 8

Meszaros, P., \& Nagel, W. 1985, ApJ, 298, 147

Mihara, T., Makishima, K., \& Nagase, F. 1998, AdSpR, 22, 987

Monard, L. A. G. 2010, CBET, 2289, 1

Mushtukov, A. A., Suleimanov, V. F., Tsygankov, S. S., \& Poutanen, J. 2015, MNRAS, 454, 2539

Pinto, C., Middleton, M. J., \& Fabian, A. C. 2016, Nat, 533, 64

Pintore, F., Zampieri, L., Stella, L., et al. 2017, ApJ, 836, 113

Poutanen, J., Lipunova, G., Fabrika, S., Butkevich, A. G., \& Abolmasov, P. 2007, MNRAS, 377, 1187

Poutanen, J., Mushtukov, A. A., Suleimanov, V. F., et al. 2013, ApJ, 777, 115

Shakura, N. I., \& Sunyaev, R. A. 1973, A\&A, 24, 337

Staubert, R., Shakura, N. I., Postnov, K., et al. 2007, A\&A, 465, L25
Steiner, J. F., Narayan, R., McClintock, J. E., \& Ebisawa, K. 2009, PASP, 121,1279

Strüder, L., Briel, U., Dennerl, K., et al. 2001, A\&A, 365, L18

Tanaka, Y. 1986, in IAU Coll. 89, Radiation Hydrodynamics in Stars and Compact Objects, ed. D. Mihalas \& K.-H. A. Winkler (Berlin: Springer), 198

Tiengo, A., Esposito, P., Mereghetti, S., et al. 2013, Nat, 500, 312

Tsygankov, S. S., Lutovinov, A. A., Churazov, E. M., \& Sunyaev, R. A. 2006, MNRAS, 371, 19

Turner, M. J. L., Abbey, A., Arnaud, M., et al. 2001, A\&A, 365, L27

Verner, D. A., Ferland, G. J., Korista, K. T., \& Yakovlev, D. G. 1996, ApJ, 465,487

Villar, V. A., Berger, E., Chornock, R., et al. 2016, ApJ, 830, 11

Vybornov, V., Klochkov, D., Gornostaev, M., et al. 2017, A\&A, 601, A126

Walton, D. J., Fuerst, F., Heida, M., et al. 2018a, arXiv:1803.04424

Walton, D. J., Fürst, F., Harrison, F. A., et al. 2018b, MNRAS, 473, 4360

Walton, D. J., Middleton, M. J., Pinto, C., et al. 2016a, ApJL, 826, L26

Walton, D. J., Tomsick, J. A., Madsen, K. K., et al. 2016b, ApJ, 826, 87

Wilms, J., Allen, A., \& McCray, R. 2000, ApJ, 542, 914 